\title{
GROUP RINGS WITH SOLVABLE $n$-ENGEL UNIT GROUPS ${ }^{1}$
}

\author{
J. L. FISHER, M. M. PARMENTER AND S. K. SEHGAL
}

\begin{abstract}
Let $K G$ be the group ring of a group $G$ over a field of characteristic $p>0, p \neq 2,3$. Suppose $G$ contains no element of order $p$ (if $p>0$ ). Group algebras $K G$ with unit group $U(K G)$ solvable and $n$-Engel are characterized.
\end{abstract}

Let $K G$ be the group ring of a group $G$ over a field $K$ of characteristic $p \geqslant 0$ and let $U(K G)$ denote its group of units. Several authors including Bateman [1], Bateman and Coleman [2], Motose and Tominaga [10] and Khripta [5] have studied the question as to when $U(K G)$ is solvable or nilpotent. Khripta in a beautiful paper [5] has proved that if $p>0$ and $G$ has a $p$-element then $U(K G)$ is nilpotent if and only if $G$ is nilpotent and the derived group $G^{\prime}$ is a finite $p$-group, settling the nonsemiprime case. This, incidently, is equivalent to saying that $K G$ is Lie nilpotent (see [11] and [14]). Khripta also has some results in her thesis on the nilpotency of $U(K G)$ in the semiprime case. We investigate when $U(K G)$ is a solvable $n$-Engel group; more precisely we prove

THEOREM. Suppose $K G$ is a group ring over a field $K$ of characteristic $p \geqslant 0$, $p \neq 2,3$. Suppose $G$ has no element of order $p$ (if $p>0$ ). Then the following are equivalent.

(i) $U(K G)$ is solvable and n-Engel.

(ii) $G$ is solvable and $m$-Engel and one of (a), (b) holds.

(a) $T(G)$, the set of torsion elements of $G$, is central in $G$.

(b) $|K|=2^{\beta}-1=p$, a Mersenne prime; $T(G)$ is abelian of exponent $\left(p^{2}-1\right)$ and for $x \in G, t \in T(G), x t \neq t x \Rightarrow x^{-1} t x=t^{p}$.

(iii) $U(K G)$ is nilpotent.

We are indebted to the referee for several useful comments.

1. Notations and definitions. For group elements $x, y$ we write the commutator $(x, y)=x y x^{-1} y^{-1}$ and

$$
(x, \underbrace{y, y, \ldots, y}_{n+1})=(x, \underbrace{y, \ldots, y}_{n}) y(x, \underbrace{y, \ldots, y}_{n})^{-1} y^{-1} .
$$

A group $H$ is $n$-Engel if it satisfies

$$
(x, \underbrace{y, \ldots, y}_{n})=1 \text { for all } x, y \in H
$$

Received by the editors December 4, 1974.

AMS (MOS) subject classifications (1970). Primary 16A26; Secondary 20F45.

Key words and phrases. Group rings, $n$-Engel, solvable.

${ }^{1}$ This work has been supported by N.R.C. Grant Nos. A-5300, A-8775 and A-7550.

(1) American Mathematical Society 1976 
and fixed $n$. Let $F$ be the multiplicative group of a field $F$. We denote by $\mathcal{E}=\mathcal{E}(\dot{F})$, the ring of endomorphisms of $\dot{F}$. We write $f^{\alpha}$ for the image of $f$ under $\alpha$ for $f \in \dot{F}, \alpha \in \mathcal{E}$. Thus $f^{\alpha+\beta}=f^{\alpha} \cdot f^{\beta}$ and $f^{\alpha \beta}=\left(f^{\alpha}\right)^{\beta}$.

By a crossed product $K\left(G, \rho_{g, h}, \alpha_{g}\right)$, we understand the set of finite sums, $\left\{\sum k_{i} \bar{g}_{i} \mid k_{i} \in K, g_{i} \in G\right\}$ where $\bar{g}_{i}$ is a symbol corresponding to $g_{i}$ and $\rho$ : $G \times G \rightarrow \dot{K}$ is a factor system and $\alpha_{g}$ is an automorphism of $K$ for each $g \in G$. Equality and addition are defined componentwise. And, for $g, h \in G$, $k \in \dot{K}, \bar{g} \cdot \bar{h}=\rho_{g, h} \overline{g h}, g k=k^{\alpha_{g} g}$ where $\rho$ and $\alpha$ are required to satisfy the necessary conditions for $K\left(G, \rho_{g, h}, \alpha_{g}\right)$ to be a ring. For details, we refer to [3].

As a special case, if we have $\alpha_{g}=I$ for all $g \in G$, we call

$$
K\left(G, \rho_{g, h}, I\right)=K^{t}(G)
$$

the twisted group ring (see [12]). If

$$
\rho_{g, h}=1 \text { for all } g, h \in G,
$$

we call $K\left(G, 1, \alpha_{g}\right)$, the skew group ring and denote it by $K_{\alpha}(G)$. And, of course, if also $\alpha_{g}=I$ for all $g \in G$, we have the (ordinary) group ring. We shall have occasion to use both skew and twisted group rings.

2. The skew group ring of an infinite cyclic group. Let $F$ be a field contained in $K G$. Suppose that $x \in G$ has infinite order, $\langle x\rangle$ is linearly independent over $F$, and that $x$ induces an automorphism $\alpha=\alpha_{x}$ of $F$ by conjugation, i.e. $\alpha: f \rightarrow x f x^{-1}=f^{\alpha}$. Then we have an isomorphic copy of the skew group ring $F_{\alpha}\langle x\rangle$ contained in $K G$. Hence $F_{\alpha}\langle x\rangle=\left\{\sum f_{i} x^{i} \mid f_{i} \in F\right\}$ where addition and equality are componentwise and $x f=f^{\alpha} x$. We investigate $F_{\alpha}\langle x\rangle$ in this section.

Lemma 2.1. For all $f \in \dot{F}$, we have

$$
(f, \underbrace{x, x, \ldots, x}_{m})=f^{(1-\alpha)^{m}} .
$$

Proof. We use induction on $m$. Notice that

$$
(f, x)=f x f^{-1} x^{-1}=f \cdot\left(f^{-1}\right)^{\alpha}=f \cdot f^{-\alpha}=f^{(1-\alpha)} .
$$

Suppose we already know that (2.2) holds for $m$; then

$$
\begin{aligned}
(f, \underbrace{x, x, \ldots, x}_{m+1}) & =f^{(1-\alpha)^{m}} x\left(f^{(1-\alpha)^{m}}\right)^{-1} x^{-1} \\
& =f^{(1-\alpha)^{m}} x f^{-(1-\alpha)^{m}} x^{-1}=f^{(1-\alpha)^{m+1}} .
\end{aligned}
$$

The lemma is proved.

Proposition 2.3. Let $F$ be an infinite field of characteristic $p \geqslant 0$ and $\alpha$ be an automorphism of finite order. Suppose that in $F_{\alpha}\langle x\rangle$ we have

$$
(f, \underbrace{x, x, \ldots, x}_{m})=1 \text { for all } f \in \dot{F} \text {. }
$$

Then $F_{\alpha}\langle x\rangle=F\langle x\rangle$, i.e. $\alpha$ is the identity automorphism.

Proof. We have by the last lemma, for all $f \in \dot{F}$, 


$$
1=f^{(1-\alpha)^{m}}=f^{\Sigma_{0}^{m}(-1)^{i}}\left(\begin{array}{c}
m \\
i
\end{array}\right) \alpha^{i} .
$$

Let $s>1$ be the order of $\alpha$. Choose a prime $q>\max (p, m)$ of the form $2 s k+1$. Then

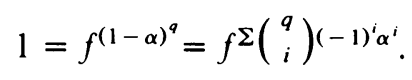

The finite automorphism group $I, \alpha, \alpha^{2}, \ldots, \alpha^{s-1}$ satisfies

$$
h\left(f, \alpha(f), \ldots, \alpha^{s-1}(f)\right)=0
$$

with

$$
\begin{aligned}
& h\left(X_{0}, X_{1}, \ldots, X_{s-1}\right) \\
& =X_{0}\left(X_{0}^{a} \cdot X_{i_{1}}^{a_{1}} \cdot X_{i_{2}}^{a_{2}} \cdots X_{i_{r}}^{a_{r}}-X_{j_{1}}^{b_{1}} \cdot X_{j_{2}}^{b_{2}} \cdots X_{j_{1}}^{b_{1}}\right), \\
& r+t=s-1,
\end{aligned}
$$

and $a=\left|\sum_{s=0}^{2 k}(-1)^{j s}\left(\begin{array}{c}q \\ j s\end{array}\right)\right|$. Since $a \equiv 1 \bmod q,(2.4)$ is a nontrivial polynomial, contradicting Artin's theorem on the algebraic independence of automorphisms of an infinite field [6, p. 228]. Hence $s=1$ and $\alpha=I$.

Proposition 2.5. Let $F$ be a finite field of $p^{a}$ elements. If $F_{\alpha}\langle x\rangle$ satisfies

$$
(f, \underbrace{x, x, \ldots, x}_{m})=1 \text { for all } f \in \dot{F},
$$

and $\alpha$ is not the identity automorphism; then, $f^{\alpha}=f^{p}$ for all $f \in \dot{F}$ and $|F|=p^{2}$, where $p$ is a Mersenne prime.

Proof. Since $f^{\alpha}=f^{p^{j}}$ for some $j<a$, we have that

$$
(f, \underbrace{x, x, \ldots, x}_{m})=f^{(1-\alpha)^{m}}=f^{\left(1-p^{j}\right)^{m}}=1 \quad \text { for all } f \in \dot{F} .
$$

Therefore, $\left(p^{a}-1\right)$ divides $\left(p^{j}-1\right)^{m}$. Hence,

$$
\text { any prime divisor of }\left(p^{a}-1\right) \text { divides }\left(p^{j}-1\right) \text {. }
$$

We claim that (2.6) implies $a=2$. Let $j$ be the smallest natural number such that (2.6) holds for a fixed $a$. Then writing, $a=j q+r$,

$$
p^{a}-1=p^{j q+r}-1=p^{r}\left(p^{j q}-1\right)+\left(p^{r}-1\right),
$$

it follows that any prime divisor of $\left(p^{a}-1\right)$ is a divisor of $\left(p^{r}-1\right)$. We may thus assume that $a=j q$. We have now that any prime divisor of $\left(p^{j}\right)^{q}-1$ is a divisor of $\left(p^{j}-1\right)$. It is easy to see (cf. [9]) that $q=2$ and $p^{j}=2^{\gamma}-1$. It follows by $[15$, p. 335] that $j=1$. Thus $a=2$. We have therefore proved that $|F|=p^{2}, p=2^{\gamma}-1$ and hence $f^{\alpha}=f^{p}$.

3. Proof of the theorem. We need the following crucial result of Lanski.

THEOREM 3.1 (LANSKI). Let $R$ be a semiprime ring which is 6-torsion free. If $U(R)$ is solvable, then all idempotents of $R$ are central.

Proof. See [7, Lemma 5] and [8, Theorem 9 and \$1]. 
We shall prove that (i) $\Rightarrow$ (ii) $\Rightarrow$ (iii) $\Rightarrow$ (i).

3.2 (i) $\Rightarrow$ (ii): Let $g$ and $h$ be elements of finite order of $G$. Since

$$
e=(1 / O(g)) \sum_{1}^{O(g)} g^{i}
$$

is an idempotent, $\langle g\rangle$ is normal by (3.1). Also $\langle h\rangle$ is normal. Thus $T_{0}=\langle g$, $h\rangle$ is a finite normal subgroup of $G$. Now,

$$
K T_{0}=\sum_{i}^{\oplus}\left(D_{i}\right)_{n_{i}}
$$

a direct sum of full matrix rings $\left(D_{i}\right)_{n_{i}}$ over division rings $D_{i}$. It follows by [4] that each $n_{i}=1$ and each $D_{i}$ is a commutative field $F_{i}$. Hence $g h=h g$. Thus $T=T(G)$, the torsion elements of $G$ form a normal abelian subgroup of $G$.

Let $x \in G, x \notin T$ and let $T_{0}$ be a finite subgroup of $T$. Suppose that $x$ does not commute with $T_{0}$ elementwise. Since every finite subgroup is normal in $G$, the skew group ring $\left(K T_{0}\right)_{\alpha}\langle x\rangle$ is contained in $K G$, where $\alpha$ is the automorphism of $K T_{0}$ induced by conjugation by $x$. Now, $K T_{0}=\Sigma^{\oplus} F_{i}$, where $F_{i}$ are fields. Also,

$$
K G \supset\left(K T_{0}\right)_{\alpha}\langle x\rangle=\left(\sum^{\oplus} F_{i}\right)_{\alpha}\langle x\rangle \simeq \sum^{\oplus}\left(F_{i}\right)_{\alpha}\langle x\rangle .
$$

The last isomorphism follows because every idempotent is central in $K G$ by (3.1) and $x F_{i} x^{-1}=F_{i}$.

We can conclude from (3.3) that the unit group of each $\left(F_{i}\right)_{\alpha}\langle x\rangle$ is $n$-Engel. Since $F_{i}$ is algebraic over $K$, it follows by Propositions 2.3 and 2.5 that $|K|=p$ or $p^{2}$, where $p$ is a Mersenne prime. If $|K|=p^{2}$ then

$$
\left|F_{i}\right|=|K| \Rightarrow F_{i}=e\left(K T_{0}\right)=e K, \quad e^{2}=e .
$$

Since every idempotent is central, $F_{i}$ commutes with $x$. Thus we have $|K|=p=2^{\beta}-1$. It remains to prove that $T_{0}^{\left(p^{2}-1\right)}=1$ and

$$
x t \neq t x, \quad t \in T_{0} \Rightarrow x^{-1} t x=t^{p} .
$$

We first make two observations. Write $T_{0}=E \times A$, where $E$ is a 2-group and $A$ is an odd group.

3.4. $A$ is central.

Let $g \in A$, then since $x^{2}$ is central, $\langle x, g\rangle /\left\langle x^{2}\right\rangle$ is a nilpotent group of order $2 \cdot O(g)$. Thus $x g x^{-1}=g x^{2 l}$ and also $x g x^{-1}=g^{i}$ as $\langle g\rangle$ is normal in $G$. Hence $x g x^{-1}=g$.

3.5. If $g$ and $h$ are nonidentity elements of $T_{0}$ then $(1-g)(1-h) \neq 0$. This is because the coefficient of identity in this product is 1 or 2 and $p \neq 2$.

Suppose that $T_{0}^{\left(p^{2}-1\right)} \neq 1$. Choose $g, h \in T_{0}$ with $h^{x} h^{-1} \neq 1$ and $g^{p^{2}-1}$ $\neq 1$. Then

$$
\pi=\left(1-g^{p^{2}-1}\right)\left(1-h^{x} h^{-1}\right) \neq 0 .
$$

Therefore, there exists an $F_{i}$ and a homomorphism

$$
\lambda: K T_{0} \rightarrow F_{i}
$$


with $\lambda(\pi) \neq 0$. Thus $\lambda(g)^{p^{2}-1} \neq 1$ and $\left|F_{i}\right|>p^{2}$. Since $\lambda\left(h^{x} h^{-1}\right) \neq 1$, we have $\lambda\left(h^{x}\right)=\lambda(h)^{x} \neq \lambda(h)$ and $F_{i}$ is not central, contradicting Proposition 2.5. We have therefore proved that $T_{0}^{\left(p^{2}-1\right)}=1$.

In order to complete the proof of the implication (i) $\Rightarrow$ (ii) it suffices to prove

$$
g \in T_{0}, \quad x g \neq g x \Rightarrow x^{-1} g x=g^{p} .
$$

We can write $g=g_{1} g_{2}, O\left(g_{1}\right)=2^{s}$ and $O\left(g_{2}\right)$ a divisor of $(p-1) / 2$. Since $g_{2}^{p}=g_{2}$ and $g_{2}$ is central due to (3.4), we have only to prove that $x^{-1} g_{1} x$ $=g_{1}^{p}$.

We may assume that $s>1$. Suppose that $K\left\langle g_{1}\right\rangle=F_{1} \oplus F_{2} \oplus \cdots,\left|F_{1}\right|$ $=p^{2}=\left|F_{2}\right|$ and $g_{1}=(\xi, \eta, \ldots), x^{-1} g_{1} x=\left(\xi^{p}, \eta, \ldots\right)$. Since $x^{-1} g_{1} x=g_{1}^{i}$, we have $p-i \equiv 0(\bmod 4)$ and $i-1 \equiv 0(\bmod 4)$ and thus $p-1 \equiv 0(\bmod$ 4) which is a contradiction. Hence $x^{-1} g x=g^{p}$.

3.8. (ii) $\Rightarrow$ (iii).

3.9. We assert that every idempotent of $K T$ is central in $K G$. If (ii)(a) holds, the assertion is trivial. So let us assume (ii)(b). Let $e=e^{2}=\sum e_{g} g$. Then $e=e^{p}=\sum e_{g} g^{p}$ and therefore $e_{g}=e_{g^{p}}$. Now $e^{x}=\sum e_{g} g^{x}=e$, since $g^{x}=g$ or $g^{p}$.

Since $G$ is $m$-Engel solvable it follows by [13, Theorem 7.36] that $G / T(G)$ is nilpotent (say of class $\leqslant c$ ). We have that either $T(G)$ is central or $|K|=p=2^{\beta}-1$ satisfying (ii)(b). We shall prove that $U(K G)$ is nilpotent of class $\leqslant(c+\beta+1)$. We may therefore assume that $G$ is finitely generated and, hence, by [13, Theorem 7.34] that $G$ is nilpotent. Therefore $T=T(G)$ is finite.

We have, $K T=\Sigma^{\oplus} F_{i}$ a finite direct sum of fields. Due to (3.9),

$$
K G=(K T)(G / T, \rho, \alpha)=\sum^{\oplus} F_{i}(G / T, \rho, \alpha) .
$$

Since $G / T$ is ordered, $U(K G)=\Pi^{\otimes} \dot{F}_{i} \cdot G / T$. It suffices to prove that $\dot{F}_{i}$. $G / T$ is nilpotent of class $\leqslant c+\beta+1$. This is clear if $\alpha$ is trivial, i.e. if $F_{i}$ and $G / T$ commute. We may therefore suppose that we have $\left|F_{i}\right|=p^{2}$, $p=2^{\beta}-1$ and we wish to prove that $F_{i} \cdot G / T$ is nilpotent of class $\leqslant c+\beta$ +1 . It is easy to see that $F_{i} \subset z_{\beta+1}$, the $(\beta+1)$ th term of the upper central series of $\left(F_{i} \cdot G / T\right)$. Since $G / T$ is nilpotent of class $\leqslant c, F_{i} \cdot G / T$ is nilpotent of class $\leqslant(c+\beta+1)$.

3.10. (iii) $\Rightarrow$ (i) is trivial.

\section{REFERENCES}

1. J. M. Bateman, On the solvability of unit groups of group algebras, Trans. Amer. Math. Soc. 157 (1971), 73-86. MR 43 \#2118.

2. J. M. Bateman and D. B. Coleman, Group algebras with nilpotent unit groups, Proc. Amer. Math. Soc. 19 (1968), 448-449. MR 36 \#238.

3. A. A. Bovdi and S. V. Mihovski, Idempotents of crossed products, Dokl. Akad. Nauk SSSR 195 (1970), 263-265 = Soviet Math. Dokl. 11 (1970), no. 6, 1439-1441. MR 42 \#7793.

4. L. K. Hua, On the multiplicative group of a field, Acad. Sinica Science Record 3 (1950), 1-6. MR 12, 584.

5. I. I. Khripta, Nilpotency of the multiplicative group of a group ring, Mat. Zametki 11 (1972), 191-200 = Math. Notes 11 (1972), no. 2, 119-124.

6. S. Lang, Algebra, Addison-Wesley, Reading, Mass., 1965. MR 33 \#5416. 
7. C. Lanski, Some remarks on rings with solvable units, Ring Theory (Proc. Conf., Park City, Utah, 1971), Academic Press, New York, 1972, pp. 235-240. MR 49 \#7311.

8. __ Subgroups and conjugates in semi-prime rings, Math. Ann. 192 (1971), 313-327. MR 44 \# 4047.

9. A. Meir and S. K. Sehgal, Canad. Math. Bull. (to appear).

10. K. Motose and H. Tominaga, Group rings with solvable unit groups, Math. J. Okayama Univ. 15 (1971/72), 37-40. MR 46 \#423.

11. I. B. S. Passi, D. S. Passman and S. K. Sehgal, Lie solvable group rings, Canad. J. Math. 25 (1973), 748-757. MR 48 \#4092.

12. D. S. Passman, Infinite group rings, Pure and Appl. Math., no. 6, Dekker, New York, 1971. MR 47 \#3500.

13. D. J. S. Robinson, Finiteness conditions and generalized soluble groups, Parts 1, 2, Ergebnisse der Mathematik und ihrer Grenzgebiete, Bände 62, 63, Springer-Verlag, New York and Berlin, 1972. MR 48 \#11314, 11315.

14. S. K. Sehgal, Lie properties in modular group algebras, Orders, Group Rings and Related Topics (Proc. Conf., Ohio State Univ., Columbus, Ohio), Lecture Notes in Math., vol. 353, Springer-Verlag, New York, 1973.

15. W. Sierpinski, Elementary theory of numbers, Parts I, II, Monografie Mat., Tom 19, 38, PWN, Warsaw, 1950, 1959; English transl., Monografie Mat., Tom 42, PWN, Warsaw, 1964. MR 31 \#116.

Department of Mathematics, University of Alberta, Edmonton, Alberta, Canada (Current address of J. L. Fisher and S. K. Sehgal)

Department of Mathematics, Memorial University of Newfoundland, St. John's, Newfoundland, Canada (Current address of M. M. Parmenter) 